\title{
EQUALIZAÇÃO AUTODIDATA: FUNDAMENTOS, NOVAS PROPOSTAS E PERSPECTIVAS
}

\author{
Carlos A. F. da Rocha ${ }^{1}$ e João M. T. Romano ${ }^{2}$ \\ ${ }^{1}$ Universidade Federal de Santa Catarina \\ LINSE-EEL-CTC-UFSC,CP.478, 88040-900 Florianópolis/SC \\ tel(048)231-9504, fax(048)2319770, e-mail:aurelio@linse.ufsc.br \\ ${ }^{2}$ Universidade Estadual de Campinas \\ Departamento de Comunicações (DECOM) -FEE-UNICAMP, \\ CP 6101, 13081-970 Campinas /SP
}

tel: (019) 239 8324, fax: (019) 239 1395, e-mail: romano@decom.fee.unicamp.br

\begin{abstract}
RE.SI NO A equalização autodidata é uma técnica utilizada para a equalização de canais de comunicação sem a ajuda da sequincra de treinamento convencional. Este trabalho fornece uma visão de síntese a cerca de três técnicas autodidatas: as Tecnica de Bussgang, as técnicas baseadas nas Estatísticas de Ordem Superior (EOS) e as Técnicas Preditivas. As úluma sio baseadas na teoria de predição linear, recentemente propostas em trabalhos anteriores. Resultados de ımuluicin comparam o desempenho das diferentes técnicas. Estes resultados mostram a potencialidade das Técnicas Preditud
\end{abstract}

IB I R I I Self-learning equalization is a technique for communication channel equalization without the aid of the uмu.d Ir.umm: ‘'yuence. This paper provides an overview about three self-learning approaches: the Bussgang Techniques, thi Ityl Ordir Statistics (HOS) Techniques and the Predictive Techniques. The latter are based on the linear prediction theor: that we have proposed in previous publications. Simulations results compare the performance of the different apprusin The results show the potentiality of the predictive techniques.

Pallı ras ( halves: "Equalização Autodidata: Fundamentos, Novas Propostas e Perspectivas”Interferência entre símbolos, equalizaçāo autodidata, algoritmos de Bussgang, estatisticas de ordem superior, técnicas preditivas.

\section{INTRODUÇÃO}

A interferência entre símbolos (IES) causada por um canal com distorção linear é o principal fator limitante no desempenho de um sistema de comunicações digitais. Basicamente, a IES é a extensão de um símbolo transmitido sobre um intervalo de tempo maior do que sa duração original. Propagação por multipercurso, como em sistema de comunicações móveis e largura de faixa limitada, como em canais telefônicos, são exemplos de fatores responsáveis pelo aparecimento da IES.

A remoção da IES é geralmente executada pelo uso de equalizadores adaptativos no receptor.

A técnica clássica utilizada para ajustar os coeficientes deste tipo de equalizador utiliza uma sequência de treinamento $d_{k}$ gerada no receptor que é a mesma sequência $a_{k}$ transmitida, a menos de um atraso $\tau$. Assim, um sinal de erro verckadeiro $e_{k}=l_{k}-d_{k}$, onde $l_{k}$ é o sinal de saída do equalizador, é formado e utilizado pelo algoritmo de adaptação a fim de levar o vetor de coeficientes $I_{k}$ do equalizador para um valor tal que os símbolos decididos $\hat{a}_{k}$ sejam corretos. Os símbolos $\hat{a}_{k}$ são obtidos na saída de um dispositivo de decisão, tendo como entrada o sinal $v_{k}$, e cujos valores de saída estão definidos no mesmo alfabeto do sinal $a_{k}$. Só após esta convergência é que a transmissão normal dos dados é iniciada e o 
erro verdadeiro é então, modificado para o erro falso: $\varepsilon_{\dot{\hbar}}=\underline{1}_{\xi}-\dot{a}_{h}$. Este modo de operação do equalizador é conhecido como hodo de decisáo direta e o erro $\varepsilon_{\text {: }}$ como eiro de decisáo direta. Nesta fase, o equalizador deve ser capaz de seguir as variações do canal e é por isso que esta é conhecida como período de rastreio o período anterior é chamado de período de treinamento. A Figura 1 ilustra um equalizador operando no modo de decisão direta.

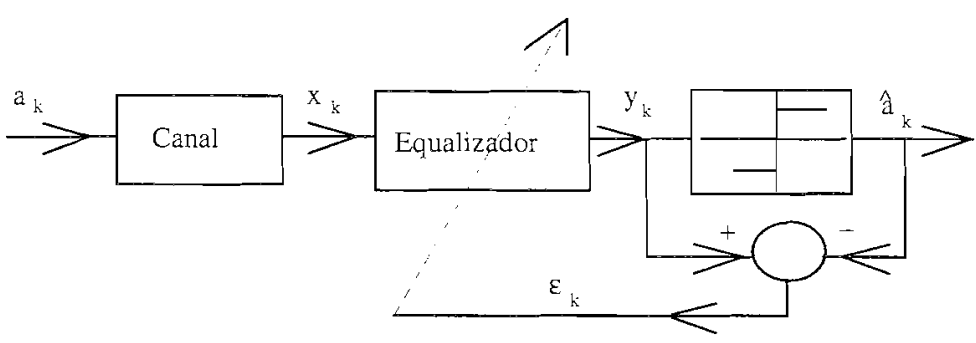

Figura1 - O modo de equalização em decisão direta (DD).

O procedimento clássico de enviar um sinal de treinamento é freqüentemente custoso, difícil e algumas vezes impossível de realizar. Alguns exemplos práticos que restringem o uso de tal sinal, são as redes multipontos para comunicação entre computadores [1], as comunicações rádio-móvel [2] e a transmissão rádio-digital na faixa de microondas [3]. Daí o interesse crescente nos chamados equalizadores cegos ou autodidatas, cujo objetivo é recuperar o sinal transmitido sem fazer uso de sequiências de treinamento.

Neste sentido, define-se desconvolução autodidata como um procedimento pelo qual se recupera um sinal aplicado à entrada de um sistema desconhecido dispondo-se apenas da sequiência de saída deste sistema e de algumas informações estatísticas sobre a entrada. A equalização de canais em transmissão digital é um caso particular de desconvolução, particularmente caracterizado pela necessidade de um processamento em tempo real, típico dos sistemas de comunicações. Assim, o problema central é a concepção de algoritmos capazes de adaptar o equalizador de modo autodidata, possibilitando a recuperação correta a partir de uma condição de "olho fechado".

As técnicas de equalização autodidatas vêm sendo intensamente discutidas na literatura nos últimos anos. O objetivo deste trabalho é prover uma visão de síntese das principais abordagens, classificadas aqui como técnicas de Bussgang [1,4-7] e técnicas baseadas em momentos de ordem superior [8-11]. Em seguida, apresentamos duas soluções baseadas na utilização de filtros de predição linear, propostos por nós em trabalhos anteriores [12-16]. Finalmente, alguns resultados de simulações comparam o desempenho das diferentes propostas e ilustram em particular o potencial das técnicas preditivas.

\section{ALGORITMOS DE BUSSGANG}

Um processo aleatório $v_{k}$ é definido como sendo de Bussgang [17] se e somente se para alguma funçāo não-linear sem memória $\Gamma($.$) , a seguinte igualdade é verificada$

$$
\mathrm{E}\left(y_{k} y_{k+m}\right)=\mathrm{E}\left(y_{k} \Gamma\left(y_{k+m}\right)\right)
$$

Sem dispor de uma réplica do sinal transmitido $a_{k}$ no receptor, a técnica de equalização autodidata dita de Bussgang utiliza uma estimativa de máxima verossimilhança $\Gamma\left(y_{h}\right)$ de $a_{k}$ baseada na suposição de uma certa distribuição de probabilidade da saída do equalizador $y_{k}$. Desta maneira, forma-se um sinal de erro $\psi\left(y_{k}\right)=y_{k}-\Gamma\left(y_{k}\right)$ utilizado para adaptar os parâmetros do filtro equalizador, através do seguinte algoritmo LMS [18] 


$$
H_{*+1}=H_{r}-\mu(y-\Gamma(y)) x_{i}
$$

onde $T_{k}=\left(x_{k}, h_{k-1}, \ldots, x_{k-1}\right)^{\mathrm{T}}$ é o vetor de sinal de entrada do equalizador e $H_{i}=\left(h_{1}, h_{2}, \ldots, h_{\mathrm{L}}\right)^{\mathrm{T}}$ é sua resposta ao impulso.

A condição de convergência na média para este algoritmo é:

$$
E\left(1, Y_{6}\right)=E(\Gamma(1)-1)
$$

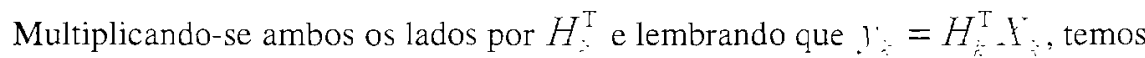

$$
E\left(y^{2}\right)=E(y-\Gamma(y))
$$

Devido a relação (4), foi considerado que a saída do equalizador l' é um processo aleatório de Bussgang [4]. Note, porém, que (4) somente satisfaz a condição de Bussgang (1) para $m=0$.

Os algoritmos de Godard [1], Stop-and-Go [5], Sato [6], Benveniste-Goursat [7], são ditos de Bussgang pois satisfazem a

\begin{tabular}{|c|c|}
\hline Algoritmo & Função de erro $\psi(y)$ \\
\hline $\mathrm{DD}$ & $\varepsilon_{k}=y_{k}-\hat{a}_{k}$ \\
\hline Sato & $\varepsilon^{\mathrm{S}}=y_{;}-\gamma \operatorname{sgn}\left(y_{k}\right), \gamma=\mathrm{E}\left(\left|a_{k}\right|^{2}\right) / \mathrm{E}\left(\left|a_{k}\right|\right)$ \\
\hline Stop-and-Go & $\because x-f \dot{a}_{k}, f=0$ ou 1 \\
\hline Benveniste-Goursat & $\kappa_{1} \cdot \varepsilon_{\mathrm{k}}+\kappa_{2} \cdot \varepsilon_{\mathrm{k}} \mid \varepsilon^{\mathrm{S}}, \kappa_{1}, \kappa_{2}=$ const. \\
\hline Godard $\left(\mathrm{CMA}^{2,2}\right)$ & $\therefore\left(\left|a^{2}\right|^{2}-\mathrm{R}_{2}\right), \mathrm{R}_{2}=\mathrm{E}\left(\left|a_{i}\right|^{4}\right) / \mathrm{E}\left(\left|a_{i}\right|^{2}\right)$ \\
\hline
\end{tabular}
condição de convergência dada pela equação (4). A Tabela I resume as funções de erro para estes algoritmos.

Tabela I - Funções de erro dos principais algoritmos de Bussgang

Os algoritmos de Bussgang são globalmente convergentes para um equalizador de comprimento infinito como provado em Beveniste et al. [19]. o qual fornece as condições suficientes para a convergência. Infelizmente, para equalizadores de comprimento finito a convergência global dos algoritmos de Bussgang não é garantida. A função custo desses algoritmos operando com tais equalizadores é não-convexa: portanto, podem ter mínimos falsos [18].

Vamos, agora, determinar o estimador de máxima verossimilhança $\Gamma($.) para uma constelação M-PAM. Para este fim, considere o modelo de equalização autodidata abaixo:

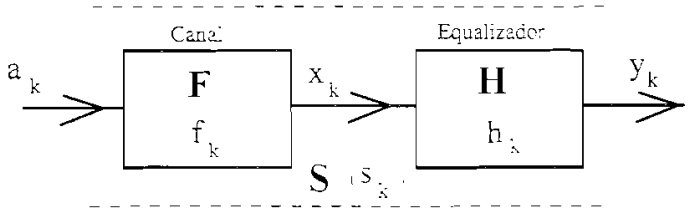

Figura 2 - Modelo de equalizaçāo autodidata

Então, podemos escrever a saída do equalizador como: 


$$
y_{k}=\sum_{i=0}^{\infty} s_{i} a_{k-1}
$$

ou

$$
y_{k=}=s_{\tau} a_{k-\tau}+\sum_{\substack{i=0 \\ i \neq \tau}}^{\infty} s_{i} a_{k-i}
$$

onde $\left|s_{\tau}\right|=\max _{k}\left|s_{k}\right|$ é o termo de maior magnitude da sequência $s_{k}$ e

$$
\eta_{k}=\sum_{\substack{i=0 \\ i \neq \tau}}^{\infty} s_{i} a_{k-i}
$$

é o chamado ruído convolucional representando a IES que resulta da inversão aproximada do canal.

Se a distribuição de probabilidade de $\eta_{k}$ é conhecida, então o estimador de máxima verossimilhança (MLE ${ }^{1}$ ) de $a_{?_{-}-}$é

$$
\left[\hat{a}_{k-\tau}\right]^{\mathrm{ML}}=\arg _{\mathrm{a}} \max p_{y_{k} / a_{k-\tau}}\left(y_{k} / a\right)
$$

onde $p_{y_{k} / a_{k-\tau}}\left(. /\right.$ ) é a distribuição condicional da saída do equalizador dada a entrada $a_{k-\tau}$. Assumindo-se que $\eta_{k}$ é um ruído branco, Gaussiano e independente de $a_{k-\tau}$ [20], o MLE torna-se simplesmente o estimador de variância mínima [21].

$$
\left[\hat{a}_{k-\tau}\right] \equiv \Gamma\left(y_{k}\right)=\mathrm{E}\left(a_{k-\tau} / y_{k}\right)
$$

Pode-se demonstrar [18] que para uma seqüência $a_{k}$ com amostras independente e identicamente distribuidas (i.i.d.) pertencente a uma constelação il-PAM, o estimador é dado por :

$$
\Gamma\left(y_{k}\right)=\frac{\left.\sum_{i=1}^{i / 2}(2 i-1) \exp -\frac{s_{\tau}^{2}(2 i-1)^{2}}{2 \sigma_{\eta}^{2}}\right] \operatorname{senh}\left[\frac{s_{\tau}(2 i-1)}{\sigma_{\eta}^{2}} y_{i}\right]}{\sum_{i=1}^{i / 2} \exp \left[-\frac{s_{\tau}^{2}(2 i-1)^{2}}{2 \sigma_{\eta}^{2}}\right] \cosh \left[\frac{s_{\tau}(2 i-1)}{\sigma_{\eta}^{2}} y_{k}\right]}
$$

Esta função é determinada pelo tamanho 1 / do alfabeto $\mathbf{A}$, constituido pelo conjunto de valores assumidos pelos símbolos $a_{k}$, pela variância do ruído convolucional $\sigma_{\eta_{i}}^{2}$ e pela relação sinal-ruído (RSR) na saída do equalizador, dada por

$$
\operatorname{RSR}=\frac{\mathrm{E}\left[\left(s_{\tau} a_{-\tau}\right)^{2}\right]}{\sigma_{\eta}^{2}}=\frac{s_{\tau}^{2}\left(M^{2}-1\right)}{3 \sigma_{\eta}^{2}}
$$

\footnotetext{
${ }^{1}$ do inglês MLE - maximum likelihood estimator
} 

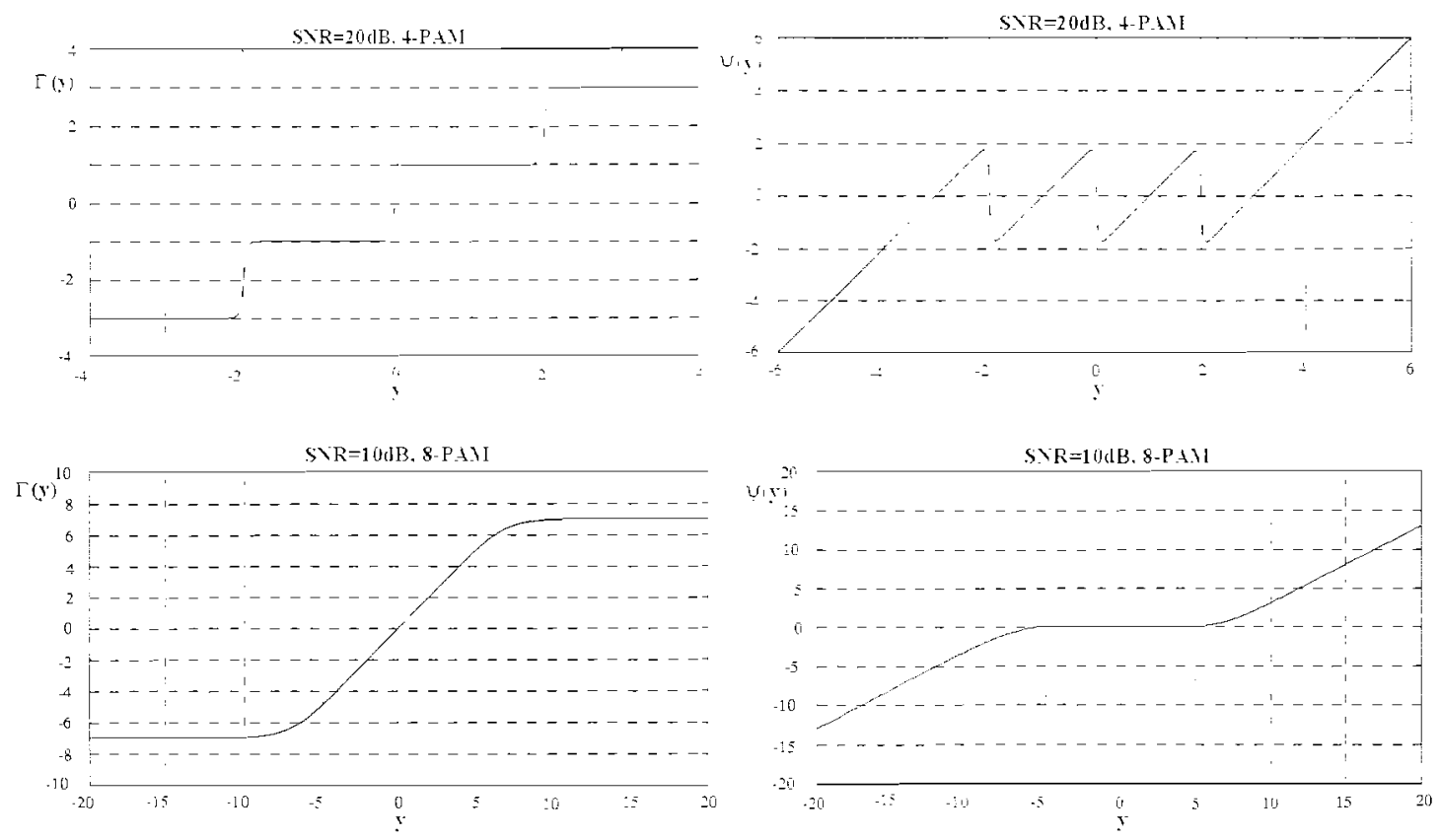

Figura - 3 - O estimador não-linear sem memória $\Gamma(y)$ e a função de erro $\psi(y)$ para os algoritmos de Bussgang sob uma entrada 4-PAM e 8-PAM

$\therefore$ Figura 3 mostra algumas funções de erro $\psi(y)=y-\Gamma(y)$ sob diferentes condições para as quais o estimador sem memória $\Gamma(y)$ é derivado.

Dessas figuras podemos concluir o seguinte:

- Quando a relação sinal-ruído (RSR) é elevada, o algoritmo de Bussgang tende ao algoritmo de decisão direta.

- É interessante notar que para uma RSR baixa e $I \geq 8$ a característica entrada-saída do estimador não-linear é bem aproximada por uma não-linearidade do tipo sigmóide:

$$
\hat{a}_{k-\tau}=\alpha \frac{1-\mathrm{e}^{-\beta y_{k}}}{1+\mathrm{e}^{-\beta y_{k}}}=\alpha \tanh \left(\frac{\beta y_{k}}{2}\right)
$$

Uma não-linearidade do tipo sigmóide é usada no projeto de redes neurais. Em particular, a combinação de um filtro transversal e uma não-linearidade sigmóide constitui um neurônio. Assim, podemos visualizar o equalizador cego como sendo essencialmente um neurônio [20]. A utilização das técnicas de redes neuronais como mais uma linha de pesquisa na busca de uma solução ótima para o problema de equalização autodidata já apresenta alguns resultados como os propostos em [22].

\section{EQUALIZAÇÃO CEGA DE CANAIS DE FASE NÃO-MÍNIMA USANDO ESTATÍSTICAS DE ORDEM SUPERIOR (EOS).}

Um considerável esforço de pesquisas tem sido realizado na área de estimação e equalização autodidata utilizando as estatísticas (cumulantes) de ordem superior do sinal recebido $x_{k}$ [8-11]. As razões para esse interesse nos cumulantes ou nas suas transformadas de Fourier conhecidas como poliespectros, são basicamente três:

1 - Os poliespectros tem a capacidade de indentificar canais de comunicação de fase não-mínima da sequência de saída devido a sua característica em preservar as informações de fase e magnitude do canal.

2 - Todos os poliespectros de um processo gaussiano de ordem maior do que 2 são nulos. Consequientemente, esta técnica não será afetada por ruídos aditivos gaussiano que podem estar presentes nos sistemas de comunicaçōes. Este é, também, o motivo que impõe aos símbolos transmitidos uma função densidade de probabilidade não gaussiana. 
3 - Os equalizadores autodidatas baseados nos poliespectros garantem convergência global para a solução desejada.

Apesar das vantagens listadas acima, as técnicas de equalização autodidatas baseadas nas EOS's necessitam realizar estimações dos cumulantes, o que aumenta consideravelmente a complexidade computacional, além de exigir estimadores confiavelmente não polarizados e um período reservado para suas inicializações, onde não é realizado o ajuste do equalizador [3].

Consideremos um canal de fase não-mínima com resposta ao impulso $f_{k}$ excitado pela seqüência i.i.d. de média nula $a_{k}$. A relação entre os cumulantes de ordem superior da saída do canal $x_{k}$ e da seqüência i. i. d. de entrada é [23]:

$$
C_{n, \ldots}\left(\tau_{1}, \tau_{2}, \ldots, \tau_{n-1}\right)=\gamma_{n, i} \sum_{l} f_{l} f_{l+\tau_{1}} \ldots f_{l+\tau_{n-1}}
$$

onde $\gamma_{n, a}$ é o cumulante de n-ésima ordem se $a_{k}$. O poliespectro é a transformada de Fourier de (n-1)-ésima ordem de $C_{n, x}^{r}\left(\tau_{1}, \tau_{2}, \ldots, \tau_{n-1}\right)$ e é dado por

$$
S_{n, l}\left(\mathrm{e}^{j \omega_{1}}, \ldots, \mathrm{e}^{j \omega_{n-1}}\right)=\sum_{\tau_{1}=\infty}^{-\infty} \ldots \sum_{\tau_{j,-1}=\infty}^{-\infty}\left(\gamma_{n, a} \sum_{i} f_{l} f_{l+\tau_{1}} \ldots f_{i+\tau_{n-1}}\right) \exp \left(-j \sum_{i=1}^{n} \omega_{i} \tau_{l}\right)
$$

Após algumas manipulação algébricas, obtém-se a partir da expressão (14)

$$
S_{n, x}\left(\mathrm{e}^{j \omega_{1}}, \ldots, \mathrm{e}^{j \omega_{1,-1}}\right)=\gamma_{n, l} F\left(\mathrm{e}^{j \omega_{1}}\right) F\left(\mathrm{e}^{j \omega_{2}}\right) \ldots F\left(\mathrm{e}^{-j\left(\omega_{1}+\omega_{2}+\ldots+\omega_{1,-1}\right)}\right)
$$

Assim, por exemplo, o Biespectro de $\left\{x_{k}\right\}$ é:

$$
S_{3, \cdots}\left(\mathrm{e}^{\prime \omega_{1}}, \mathrm{e}^{j \omega_{2}}\right)=\gamma_{3_{s},} F\left(\mathrm{e}^{j \omega_{1}}\right) F\left(\mathrm{e}^{j \omega_{2}}\right) F\left(\mathrm{e}^{-j\left(\omega_{1}+\omega_{2}\right)}\right)
$$

onde $\gamma_{3, a}=E\left(a_{k}^{3}\right)$. Desta forma, a fase e a magitude de $S_{3, x}\left(\mathrm{e}^{\omega_{1}}, \mathrm{e}^{j \omega_{2}}\right)$ estão associadas diretamente à fase e à magnitude de $F\left(\mathrm{e}^{j \omega}\right)$, e temos então:

$$
\begin{aligned}
& \operatorname{Fase}\left[S_{3, \cdots}\left(\mathrm{e}^{j \omega_{1}}, \mathrm{e}^{j \omega_{2}}\right)\right]=\operatorname{Fase}\left[F\left(\mathrm{e}^{j \omega_{1}}\right)\right]+\operatorname{Fase}\left[F\left(\mathrm{e}^{j \omega_{2}}\right)\right]+\operatorname{Fase}\left[F\left(\mathrm{e}^{-j\left(\omega_{1}+\omega_{2}\right)}\right)\right] \\
& \log \left|S_{3, x}\left(\mathrm{e}^{\jmath \omega_{1}}, \mathrm{e}^{j \omega_{2}}\right)\right|=\log \left|\gamma_{3, a}\right|+\log \left|F\left(\mathrm{e}^{j \omega_{1}}\right)\right|+\log \left|F\left(\mathrm{e}^{j \omega_{2}}\right)\right|+\log \left|F\left(\mathrm{e}^{-j\left(\omega_{1}+\omega_{2}\right)}\right)\right|
\end{aligned}
$$

Existem diversos métodos $[9,11]$ para extrair as informações de fase e magnitude do canal desconhecido a partir do Biespectro. A extensão para um poliespectro qualquer é direta. Lembre da necessidade do dado de entrada $\boldsymbol{c}_{k}$. ser nãoGaussiano, pois caso contrário todos os poliespectros $(n>2)$ serão identicamente nulos.

Em [24] Shalvi e Weinstein demonstraram que a condição suficiente para a equalização cega de um canal de fase nãomínima sem zeros sobre a CRU é que a variância e algum cumulante de ordem superior ( $\left(_{n, y}^{*}, \forall n>2\right)$ não nulo de $y_{\text {;: }}$ seja igual aos da seqüência $a_{k}$. Esta técnica se sobrepõe às demais que utilizam EOS, principalmente, pela simplicidade do critério de otimização utilizado.

\section{AS TÉCNICAS PREDITIVAS}

Uma proposta alternativa para se chegar a algoritmos eficientes de equalização autodidata consiste em se utilizar os princípios de predição linear [20]. Sabe-se que a minimização do erro quadrático médio de predição de um sinal a partir de seus valores passados corresponde a aplicar o princípio da ortogonalidade entre o erro de estimação e essas amostras passadas. A partir daí, é fácil mostrar que o próprio erro de predição tende a ser um sinal descorrelacionado na medida em que aumentamos a ordem do filtro preditor [25]. 
A mesma propriedade se verifica quando se trata da operação de retropredição, que consiste em estimar uma determinada amostra passada a partir de valores conhecidos das amostras subsequentes. Assim, é um resultado conhecido [20] o fato de que tanto o preditor como o retropreditor tendem a ser filtros "branqueadores", sendo que o primeiro é necessáriamente de fase mínima e o segundo de fase máxima.

Essas propriedades são de grande interesse para o problema aqui tratado uma vez que se supõe que a seqüência $a_{k}$ transmitida é i.i.d. e, portanto, o equalizador será também necessariamente um filtro branqueador.

Entretanto, o preditor e o retropreditor fornecem uma solução de fase mínima e máxima, respectivamente, enquanto que æ.ralmente o canal é de fase mista. Em outras palavras, os filtros preditores aplicados isoladamente fornecem uma sequiência de saída branca mas diferente da seqüência transmitida $a_{i}$, ou ainda, equalizam a resposta em magnitude do canal mas não sua resposta de fase.

Surge então, numa primeira abordagem. a idéia da utilização conjunta do preditor e do retropreditor para se chegar à equalização correta como veremos a seguir. O modelo FIR de um canal de fase não-mínima pode ser representado como uma cascata de três sistemas lineares.

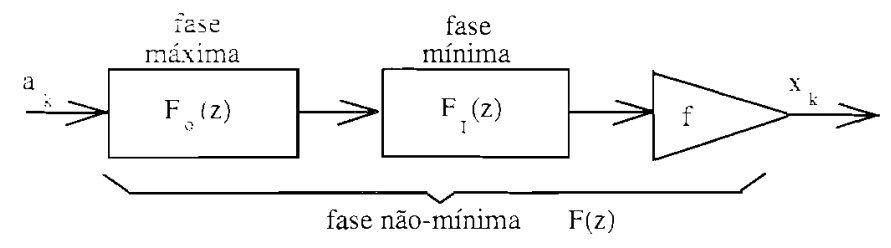

Figura 4 - Decomposição de um canal fase não-mínima

Bascidu nesta decomposição em, [12,13] propos-se uma estrutura de equalização autodidata como uma cascata de um recropreditor (R), um preditor $(\mathbf{P})$ e um $\mathbf{C A G}$ complexo $(\mathbf{G})$ ilustrada na Figura 5. O retropreditor $\mathbf{R}$ compensa a parte de fas maxuma, o preditor $\mathbf{P}$ a parte de fase mínima e o CAG G ajusta o ganho de maneira que a potência do sinal de saída 心'. I mplimınıụ̄o é irrelevante. Contudo, quando $\mathbf{P}$ for IIR, existe a opção de se incluir o dispositivo de decisão na malha de redlımbntaciu de $\mathbf{P}$ afim de suprimir ruídos. Desta forma, este sistema assemelha-se ao clássico equalizador realimentado i...: w

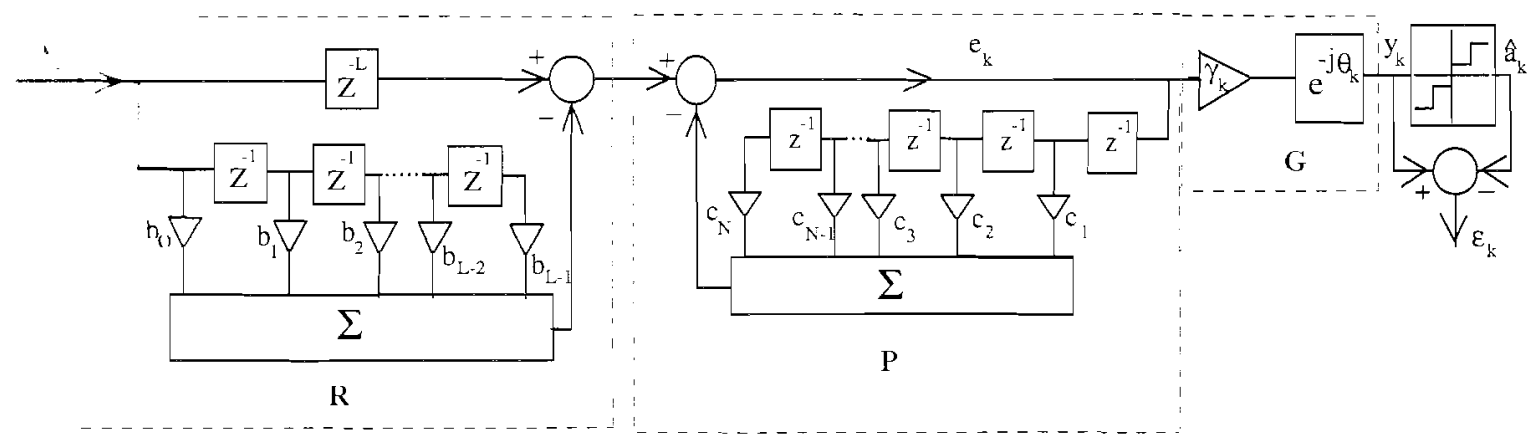

Figura 5 - O equalizador como uma cascata de um retropreditor e um preditor e um ganho complexo.

Em 1987 Macchi e Gu [26] utilizaram uma estrutura semelhante àquela da Figura 5. A adaptação dos coeficientes do preditor e do retropreditor foi realizada utilizando apenas critérios de branqueamento. Isto limitou a aplicação desta técnica a canais com características predominantemente de fase mínima ou de fase máxima. Em [12,13] foi proposto um novo critério de otimização aplicável a qualquer canal sem zeros sobre a CRU. O novo critério envolve, apenas, minimizações de erros quadráticos médios, de predição e de decisão direta, o que assegura um ótimo desempenho em termos de velocidade de convergência quando comparados a outros métodos. Além disso, esta nova técnica de equalização autodidata é robusta o suficiente para equalizar canais que provocam elevadas IES, tal como aquele de [27], onde outras técnicas falham [13]. 
Consideremos, então, o vetor $H=\left(B^{T}, C^{T}\right)^{T}$ contendo os $\mathrm{L}$ coeficientes de $\mathbf{R}$ e os $\mathrm{N}$ coeficientes de $\mathbf{P}$. O novo critério proposto em $[12,13]$ é dado por

$$
J_{\alpha}(H)=\alpha \gamma^{2} J_{P}(H)+(1-\alpha) J_{\varepsilon}(H)
$$

onde a minimização da componente $J_{P}(H)=\mathrm{E}\left(\left|e_{k}\right|^{2}\right)$ implica no branqueamento da seqüiência $e_{k}$ e da saída $y_{k}=\gamma \mathrm{e}^{j \theta} e_{k}$. Por sua vez, a componente $J_{\varepsilon}(H)=\mathrm{E}\left(\left|\varepsilon_{k}\right|^{2}\right)$ é minimizada quando a saída $y_{k}$ se identifica com $a_{k}$. O fator $\gamma^{2}$ assegura a homogeneidade do critério e $\alpha$ é inicialmente próximo de 1 , diminuindo progressivamente para ser finalmente próximo de zero. A regra adotada para $\alpha$ foi: $\alpha=\alpha_{k}=\left(\tanh \left|\varepsilon_{i-1}\right|\right)^{2}$. Assim, o critério (18) passa gradualmente de um critério de branqueamento para o critério clássico de equalização adaptativa.

Os algoritmos LMS para a adaptação dos parâmetros de $\mathbf{R}$ e $\mathbf{P}$ são obtidos a partir de (18) e são dados por [25]:

$$
B_{k}=B_{k-1}-\mu \varepsilon^{\alpha}\left(y_{k}\right) \gamma \mathrm{e}^{j \theta_{k-1}} \Xi_{k}^{*}
$$

e

$$
C_{k}=C_{k-1}-\lambda \varepsilon^{\alpha}\left(y_{k}\right) Y_{k}^{*},
$$

onde

$$
\begin{aligned}
& B=\left(b_{0}, b_{1}, \ldots, b_{L-1}\right)^{\mathrm{T}}, \quad C=\left(c_{1}, c_{2}, \ldots, c_{1}\right)^{\mathrm{T}}, \quad Y_{k}=\left(l_{k-1}^{\prime}, \ldots, y_{k-1}\right)^{\mathrm{T}}, \quad \Xi_{k}^{*}=-\left(\xi_{k-L+1}^{*} \ldots \xi_{k}^{*}\right)^{\mathrm{T}}, \\
& \xi_{k}=x_{k}-\sum_{i=1}^{1} c_{i} \xi_{k-1} \text { e onde a função } \varepsilon^{\alpha}(y) \text { é definida como: }
\end{aligned}
$$

$$
\varepsilon^{\alpha}(y) \stackrel{\Delta}{=} \alpha y-(1-\alpha) \varepsilon(y)
$$

O algoritmo dado pela equação (20) é conhecido como algorimo cle regressão pseudo-linear [28] porque o sinal de saída do preditor é uma função não-linear dos seus coeficientes e na dedução do algoritmo, supõe-se que o vetor $I_{k}$ (regressão) não depende dos coeficientes $c_{i}$. A estabilidade deste algoritmo aplicado a um filtro de predição IIR está demonstrado em [29]. O ganho $\gamma$ é ajustado por um simples controle automático de ganho (CAG)

$$
G_{k}=G_{k-1}+\eta\left(E\left(\left|\mathrm{a}_{\mathrm{k}}\right|^{2}\right)-\left|y_{k}\right|^{2}\right), \gamma_{\mathrm{k}}=\sqrt{\left|G_{k}\right|}, \eta>0
$$

com o qual $y_{i}$ deverá ter a mesma potência de $a_{k}$, após a convergência de (22). Para $\theta$, o algoritmo do gradiente estocástico de $J_{\alpha}(H)$ produz

$$
\theta_{k}=\theta_{k-1}+v\left(1-\alpha_{k-1}\right) \operatorname{Im}\left(\varepsilon_{k} v_{k}^{*}\right), \quad v>0
$$

Dentre as técnicas preditivas, uma outra abordagem pode ser feita a partir de uma decomposição alternativa do canal $F(z)$, tal como ilustrada na Figura 7. 


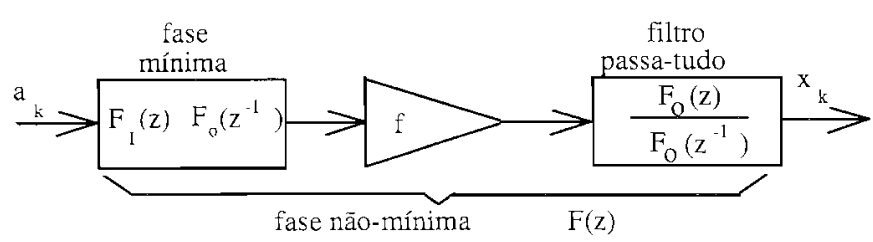

Figura 7 - Fatorização alternativa do canal de fase não-mínima $F(z)$

O equalizador ótimo, na ausência de ruídos. deverá implementar a inversão do canal $F(z)$ a menos de um retardo. Sua função de transferência é dada por.

$$
E(z)=z^{-\delta} F^{-1}(z)=H(z) \cdot g \cdot P(z)
$$

onde $P(z)=\left[F_{I}(z) F_{0}\left(z^{-1}\right)\right]^{-1}, H(z)=z^{-\delta}\left[\frac{F_{0}(z)}{F_{0}\left(z^{-1}\right)}\right]^{-1}$ e $g=f^{-1}$, e onde o retardo deve ser tal que $H(z)$ seja estável e causal. Dessa forma, a expressão (24) representa um equalizador $E(z)$ através de uma cascata de três filtros lineares: um preditor $\mathbf{P}$, um CAG $\mathbf{G}$ e um filtro passa-tudo $\mathbf{H}$ que funciona como um equalizador de fase.

Como vimos anteriormente, $P(z)$ é um filtro preditor que ao branquear o seu sinal de saída, funciona como um equalizador da resposta em magnitude do canal $F(z)$.

Desta maneira, foi proposto em [14] a estrutura de equalização autodidata mostrada na Figura 8.

Assim, enquanto o preditor $\mathbf{P}$ é responsável pela equalização de magnitude do canal, o filtro não-linear IIR $\mathbf{H}$ equaliza a fase do canal equivalente, composto pelo canal propriamente dito mais o preditor.

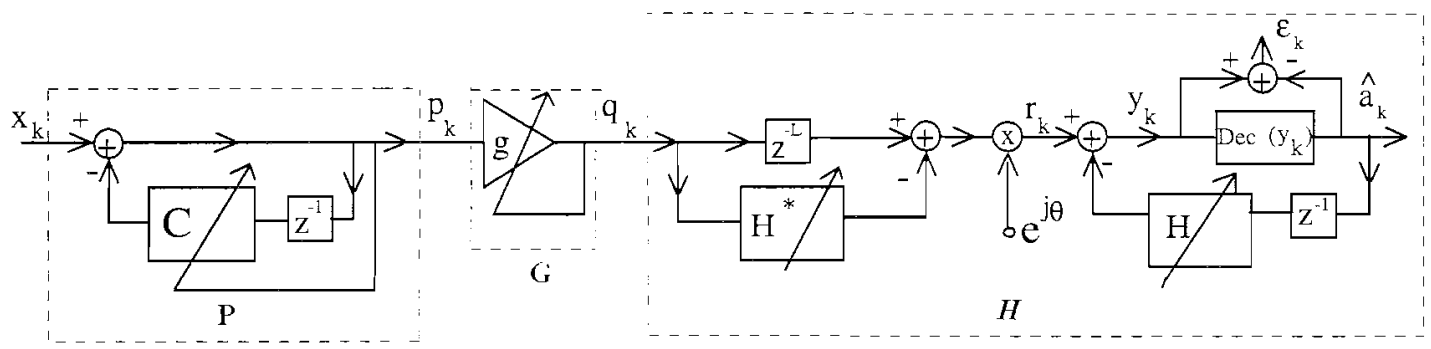

Figura 8 - Equalizador autodidata como uma cascata de um preditor IIR, um ganho e um filtro IIR não-linear.

A minimização da potência do erro $\mathrm{DD}\left(\boldsymbol{\varepsilon}_{k} \rightarrow 0\right)$ transforma o filtro $\mathbf{H}$ em um filtro passa-tudo linear. É esta linearização que assegura uma equalização correta da fase do canal passa-tudo equivalente. A demonstração desta afirmativa é simples e é dada a seguir.

Note que $\varepsilon_{k}=0$ implica em que os símbolos de saída do equalizador $v_{k}$ pertença ao alfabeto dos símbolos transmitidos A. Para esta condição o equalizador de fase não-linear $\mathbf{H}$ se reduz a um filtro passa-tudo linear, de maneira que o conjunto FoPoGoH é também passa-tudo. Temos então que:

$$
y_{k}=\mathrm{e}^{j \varphi} \frac{q^{-\underline{Q}}+\sum_{i=1}^{Q} s_{i}^{*} q^{-\underline{Q}+i}}{1+\sum_{i=1}^{Q} s_{i} q^{-t}} a_{i}
$$

onde $Q$ é ordem do filtro total equivalente. É evidente que, para $y_{k} \in \mathbf{A}$, é necessário que $s_{i}=0$ e $\varphi=\kappa \pi / 2, \kappa=0,1,2, \ldots$ o que nos conduz à seguinte identidade: 


$$
y_{k}=\mathrm{e}^{1 \varphi} a_{k-\underline{Q}},
$$

que corresponde ao canal equalizado, a menos do retardo $Q$ e da ambiguidade de fase proporcional a $\pi / 2$. Para $\kappa$ impar, esta ambiguidade pode ser resolvida através da utilização de uma modulação diferencial.

Os algoritmos de adaptação dos três blocos que constituem o equalizador da Figura 8 podem ser encontrado em [15,25] e são dados por:

$$
\begin{gathered}
C_{k}=C_{k-1}+\mu p_{k} P_{k-1}^{*}, \mu>0 \\
G_{k}=G_{k-1}+\rho\left(\mathrm{E}\left(\left|\mathrm{a}_{\mathrm{k}}\right|^{2}\right)-\left|q_{k}\right|^{2}\right), g_{\mathrm{k}}=\sqrt{\left|G_{k}\right|}, \rho>0 \\
H_{k+1}=H_{k}-\vartheta \Psi_{k}, \quad \vartheta>0
\end{gathered}
$$

onde $P_{k}=\left(p_{k-1}, p_{k-2}, \ldots, p_{k-1}\right)^{\mathrm{T}}, \Psi_{k}=\left(\psi_{k}^{1}, \ldots \psi_{k}^{L}\right)^{\mathrm{T}}$ e onde

$$
\psi_{k}^{\prime}=\varepsilon_{k}^{*} \mathrm{e}^{\mathrm{j} \theta_{\mathrm{k}}} q_{k-L+j}-\varepsilon_{\mathrm{k}} \hat{a}_{k-j}^{*}, \quad \mathrm{j}=1, \ldots, L
$$

O algoritmo de adaptação para a recuperação da fase da portadora é dado por:

$$
\theta_{k+\mathrm{I}}=\theta_{k}+v \operatorname{Im}\left(\varepsilon_{k}^{*} q_{k}\right), v>0
$$

Diversos resultados de simulações demonstraram uma maior eficiência deste equalizador quando comparado a outras técnicas de equalização autodidata, como ilustra a Figura 9 [14].

Finalmente, no que diz respeito a unimodalidade das técnicas autodidatas acima, uma análise detalhada é realizadas em [25]. Tal análise baseia-se na hipótese de que a seqüência $y_{k}$ é gaussiana nas condições de olho fechado, o que é razoável para sinais multiníveis. Com isto, foi demonstrado que a estrutura proposta, associada ao critério DD é unimodal, o que é confirmado através dos resultados de simulações.

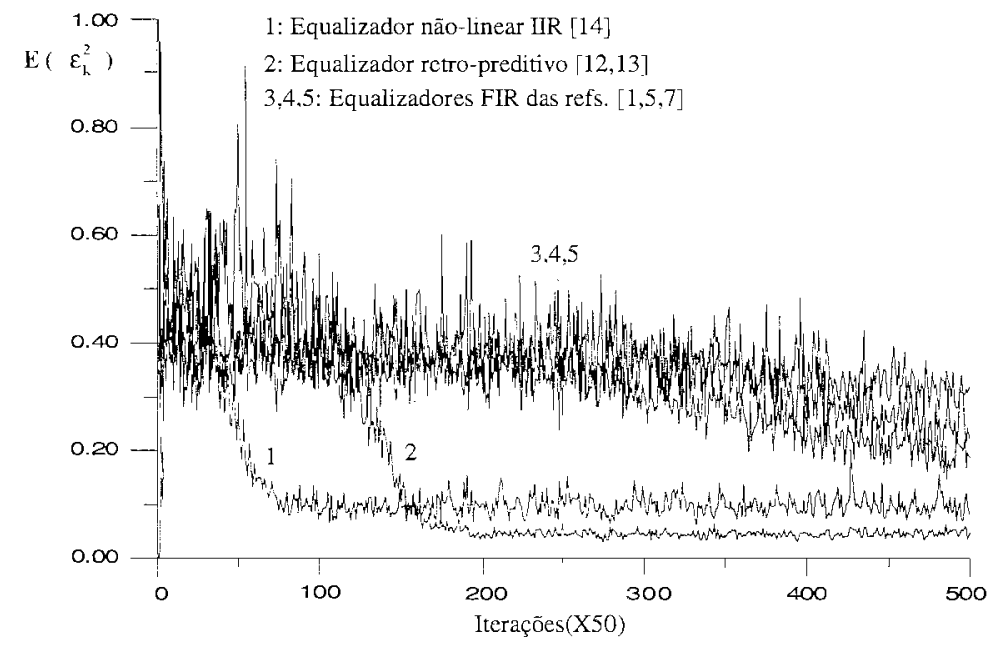

Figura 9 - Comparação de diversas técnicas de equalização autodidatas

\section{CONCLUSÕES}

Uma síntese das técnicas mais importantes para equalização autodidata foi apresentada neste trabalho, onde se procurou esclarecer o "estado da arte" no assunto e os principais fundamentos, tanto matemáticos como conceituais, das diferentes soluções propostas. Duas novas propostas, aqui classificadas como técnicas preditivas, foram também abordadas. 
Os algoritmos decorrentes da teoria de Bussgang são os que receberam um tratamento mais intenso na literatura. Entretanto, a baixa velocidade de convergência e a possível presença de mínimos locais constituem ainda um fator limitante na sua utilização. Por outro lado. apresentam baixa complexidade computacional, o que não é o caso das :-..-as que exigem uma estimação das estatísticas de ordem superior dos sinais de entrada do equalizador.

As técnicas baseadas em predição linear apresentam resultados bastante animadores no que diz respeito à velocidade de convergência, sem que isso implique em aumento da complexidade. A estrutura não-linear na Figura 8 possibilita ainda a equalização de canais com nulos espectrais, situação onde os equalizadores lineares não obtêm sucesso. Por outro lado, torna-se necessário uma futura análise dessas estruturas em meio ruidoso, onde a adaptação dos filtros IIR utilizados pode se tornar delicada. Sobretudo, percebe-se a dificuldade de apontar alguma solução que tenha um desempenho naturalmente superior em todas as situações práticas que se pode encontrar.

Finalmente, é importante reconhecer que as três técnicas de equalização autodidata abordadas neste trabalho não são exaustivas. Novas técnicas são constantemente propostas na literatura. Podemos, no mínimo, mencionar duas delas: a que utiliza a característica cicloestacionária exibida pelo sinal analógico $x(t)$ da saída do canal ou do sinal $x(t)$ amostrado a uma taxa superior à de símbolos [30] e as que utilizam as técnicas de redes neuronais [22]. O aprofundamento nessas técnicas mais recentes e nos problemas que delas decorrem abre interessantes perspectivas para trabalhos futuros.

\section{BIBLIOGRAFIA}

[1] D. N. Godard, Self-Recorering Equalizalion and Carrier Tracting in Two-Dimensional Dala Commmnication Sistems, IEEE Treans. on Communication, vol.COM-28, No.11, pg.1867-1875, Nov.1980

[2] K. Hilal, Algorithnes Acceleres d'Egalisation Adaptative Autodidacte: Application au Canal Radio-1 lobile, Tese de Doutorado, L'Ecole Nationale Supérieure des Télécommunications, Paris, França, 1993

[3] J. C. Mota, Equalização -tdaptativa em Sistemas Rádio-Digitais: das Técnicas em Freqüencia à Teoria da Desconnolução Alutodidata. Tese de Doutorado, DECOM/FEE/UNICAMP, 1992

[4] S. Bellini, Bussgang Techniques for Blind Decomolution and Equalization. em Blind Deconvolution, S. Haykin Ed., Prentice-Hall Information and System Sciences Series, pp.8-59, 1994

[5] G. Picchi e G. Prati, Blind Equalization and Carrier Recoverv L Sing a "Slop-and-Go" Decision-Directed Algorithm, IEEE Trans. on Communication, vol.COM-35, No.9, pp.877-887, Sept.1987

[6] Y. Sato, A Method of Self-Recovering Equalization for Multilevel Amplitude-1 Lodulation. IEEE Trans. on Communication, vol.Com-23, pp.679-682, June 1975

7] e M. Goursat A. Benveniste, Blind Equalizers, IEEE Trans. on Communications, vol.COM-32, No.8, pg.871-883, Aug. 1984

[8] D. Hatzinakos e C. L. Nikias, Blind Equalization Based on Higher-Order Statistics (HOS), em Blind Deconvolution, S. Haykin Ed., Prentice-Hall Information and System Sciences Series, pp.181-258, 1994

-9] C. L. Nikias, ARlLt Bispectrum Approach to Vomminimum Pliase Sistem Identification. IEEE Trans. on ASSP, vol.36, No.4, pp.513-524, April 1988

$\because$ J. K. Tugnait, Blind Estimation of Digital Communication Chanlel Impulse Rexponse. IEEE Trans. on Communication, vol.42, No.2/3/4, pp.1606-1616, Feb./March/April 1994

[11] M. R. Raghuver e C. L. Nikias, Biespectrm Estimation: A Paranemic Approach. IEEE Trans. ASSP, vol.ASSP33. pp.1213-1230, Oct. 1985

[12] O. Macchi, C. A. F. da Rocha e J. M. T. Romano, Egalisation -telaptative Alutodidacle par Rétroprédiction et Prédiction, XIV GRETSI, Juan-les-Pins, France, pp.491-494, Sept.1993

[13] C. A. F. da Rocha, O. Macchi, t Tovel Self-Leaming Complex -tdapitie Recursive Equalizer IIinh Imique Uptimnm. Proc. ICASSP-94, Adelaide, New Zealand, April 1994

[14] C. A. F. da Rocha, O. Macchi e J. M. T. Romano, th tulaptive Vonimear IIR Filter for Self-Learning Equalization. IEEE International Telecommunications Symposium,

[15] C. A. F. da Rocha, O. Macchi e J. M. T. Romano, Egaliseum Idaptative tutodidacte Comme ume Cascade de Egalisenrs de lagnitude el Phase, submetido ao XV GRETSI. 
[16] C. A. F. da Rocha,, J. M. T. Romano e O. Macchi, Self-Leaming Deconvolution Lsing a Cascade of l Fagnitude and Phase Equalizers, IEEE Proc. 1995 Midwest Symposium on Circuits and Systems, August 1995, Rio de Janeiro

[17] J. J. Bussgang, Cross Correlation Funchion of Amplitude-Distorted Gaussian Signals, Technical Report 216 , MIT Research Laboratory of Electronics, Cambridge, Mass.

[18] Z. Ding, Application Aspects of Blind telaptive Equalizer in Q.11/ Data Communications, Ph.D. Thesis, Cornell University, August 1990

[19] A. Benveniste, M. Goursat e G. Ruget, Robust Identification of a . Komminimum Phase Sistem: Blind Adjustment of a Linear Equalizer in Data Communications. IEEE Trans. on Automatic Control, vol-AC.25, No.3, pp.385-398, June 1980

[20] S. Haykin, Aclaptive Filter Theorv, Prentice-Hall International, 1991

[21] B. D. O. Anderson e J. B. Moore, Optinlal Fi/lering. Prentice-Hall, New Jersey, 1979

[22] S. Mo e B. Shafai, Blind Equalizalion Lsing ITigher Omder Cumulants and Veural Venwork. IEEE Trans. on Signal Processing, vol.42, pp.3209-3217, nov. 1994

[23] J. M. Mendel., Tutorial on Iligher-Order Statistics (Spectra) in Signal Processing and System Theor:: Theorical Results and Some tpplications, Proceedings IEEE, vol.79, No.3, pg.278-305, March 1991

[24] O. Shalvi e E. Weinstein, Imiersal Methods for Blind Deconvolution, em Blind Deconvolution, S. Haykin Ed., Prentice-Hall Information and Systems Sciences Series, pp. 121-180, 1994

[25] C. A. F. da Rocha, Técnicas Preditivas para Equalização tutodidata, Tese de Doutorado (a ser concluida), Departamento de Comunicações /FEE/ UNICAMP.

[26] O. Macchi e Yi Gu, Self-Adaptive Fqualization II ith a Mixed Bachorard and Forward Prodictor, ISELDECS, Kharagpur, India, pp.437-440, Dec. 1987

[27] B. Porat e B. Friedlander, Blind Equalization of Digital Communication Chamnels Csing High-Order Moments, IEEE Trans. on Signal Processing, vol.39, No.2, pp.522-526, February 1991

[28] J. J. Shynk, Atlaptive IIR Fillering, IEEE ASSP Magazine, pp.4-21, Abril 1989

[29] O. Macchi, Adaptive Processing: The Least Mean Squares Approach IIith Applications in Transmission, John Wiley \& Sons Ltda, 1995

[30] Z. Ding. e Y. Li, On Channel Identificarion Based on Second-Order Cuctic Spectra. IEEE Trans. on Signal Processing, vol.42, No.5, pp.1260-1264, May 1994

Carlos Aurćlio F. da Rocha recebeu o grau de Engenheiro Eletrônico pela Universidade Federal do Pará (UFPa) em 1981 e o grau de Mestre em Engenharia Elétrica pela Universidade Federal de Santa Catarina (UFSC) em 1985. De janeiro a dezembro de 1993 trabalhou como pesquisador no "Laboratoire des Signaux et Systèmes" (SUPELEC - CNRS França). Atualmente está em fase de conclusão de sua tese de doutorado no Departamento de Comunicações da Faculdade de Engenharia Elétrica da UNICAMP. Desde 1983 é professor do Departamento de Engenharia Elétrica e pesquisador do Laboratório de Instrumentação Eletrônica (LINSE) da UFSC. As áreas de pesquisa de seu interesse são: processamento digital de sinais, filtragem adaptativa autodidata e aplicações de redes neurais em equalização autodidata.

João Marcos T. Romano nasceu no Rio de Janeiro em 12 de abril de 1960. Formou-se em Engenharia Elétrica na UNICAMP em 1981 e defendeu tese de Mestrado em 1984 nesta mesma instituição. A partir de 1984 esteve no "Laboratoire des Signaux et Systèmes" (CNRS - França) onde desenvolveu sua tese de Doutorado, recebendo em Dezembro de 1987 o título de "Docteur de l'Université de Paris - XI". Desde 1988, está no Departamento de Comunicações da Faculdade de Engenharia Elétrica da LNICAMP onde, em 1992, recebeu o título de Professor LivreDocente. Foi também Professor Convidado do "Conservatoire National des Arts et Métiers" em Paris, no período de Janeiro a Março de 1993. Suas áreas de interesse concentram-se em Sistemas Adaptativos e em aplicações de técnicas de Processamento Digital de Sinais em Telecomunicações. 